\title{
JUMMEC: TAKING STOCK, CONSOLIDATING AND GAINING IN STRENGTH
}

The year 2005 marked the rebirth of JUMMEC when the Editorial Board took over from its previous Editor with modest but realistic expectations. One year on, we have successfully achieved our initial expectation, that is, to encourage greater participation from our junior academics to write and publish in our very own journal, JUMMEC. As we head towards the end of 2006 and prepare to usher in 2007, we see JUMMEC consolidating and gaining in strength. Let us reflect on its past achievements and our expectations for the future.

In the bumper 2003-2005 issue, many articles written by our Faculty members were published. Among some of these articles include an article pertaining to health issues and challenges in the 2 I st century, where changes in disease pattern, environmental health, demographic impacts on health, migrant issues and health, effects of globalization on health, mental health and wellness, and equity in health care were reviewed (I). Other reviews on cardiovascular exercise testing as a valuable physiological assessment tool in research and clinical practice (2), and an overview on peripartum cardiomyopathy, where challenges that lie in diagnosing and managing this rare yet lethal disease were highlighted (3). Apart from clinical studies, epidemiological studies such as, lifestyle practices in relation to overweight and obesity were also reported in a community-based study, where risk factors, such as physical exercise, cigarette smoking, dietary habits, eating pattern, chronic diseases and family history were studied (4).

In conjunction with the centennial celebration of the University of Malaya in 2005, the Faculty of Medicine had organized the International Conference on Health Sciences: 'Partnership in Healthcare Lessons Learnt and Future Challenges'. Some international speakers spoke at the conference and their extended abstracts are published in Supplement I, 2006. Darryl M from UNESCO wrote on biomedical ethics; Khoo SK from Brisbane, Australia touched on the clinical implications on ageing (or longevity); Booker PD from Liverpool, UK wrote on the recent advances in paediatric intensive care; $\mathrm{Ng} \mathrm{CH}$ from
Melbourne, Australia described the burden of depression in the Asia Pacific region; David Hui SC from Hong Kong on obstructive sleep apnoea and cardiovascular complications; Peter Eu from Melbourne, Australia on PET radiopharmaceutical production; Kevin Tse KM from Hong Kong who gave the Hong Kong experience in the pursuit of an ideal imaging protocol in PET and PET-CT; Herman S from Indonesia who gave a current perspective on cervical cancer in Indonesia; Partha G from Singapore wrote on PET-CT clinical image interpretation of various carcinomas; David JG from Glasgow, UK dealt with the screening for colorectal cancer; Mok QQ from London, UK who deliberated on the new strategies in ventilation; Kenneth MC from Scotland, UK shared their UK solutions to the challenges of postgraduate medical education; John $\mathrm{R}$ from Toronto, Canada reviewed the recent advances in neurotology; and Jeremy DMR and Bill F from Coventry, UK who described the basic principles of the Values-based Practice: A new approach to understanding clinical interactions. And also our very own experts in their respective areas of specialty, such as, Goh KL, Lam SK, Tan CT, Azila AMN, Liam CK, Shyamala DS, Vijayan R, Chan LL, Yeoh PH, Ednin $\mathrm{H}$, and so forth, whose abstracts were highlighted in this issue. The Editorial Board would like to thank Professor Liam Chong Kin, the Scientific Chair for compiling these abstracts.

The 2006 Issue 9 (I) bears witness to the strengthening of JUMMEC in line with the research activities of the Faculty. In that issue, you will find Chia YC's Editorial on more rational prescribing (5). Among others, that issue also carried articles on the resistance to antimicrobials and its problems, steroid withdrawal in transplant renal recipients, body-fat comparison of sport players, complementary medicine, usage of a simple neural network architecture to diagnose angina and a review on the trends of maternal mortality.

In this current issue, apart from the two papers on interdisciplinary research on impaired mobility disability and rehabilitation and the breast cancer review, many case reports - Grade IV hypertensive retiono- 